\section{BMJ Open Respiratory Research}

\title{
Lung ultrasound volume sweep imaging for respiratory illness: a new horizon in expanding imaging access
}

Thomas J Marini (D) , ${ }^{1}$ Justin M Weis, ${ }^{2}$ Timothy M Baran, ${ }^{1}$ Jonah Kan, ${ }^{3}$ Steven Meng, ${ }^{3}$ Alex Yeo, ${ }^{4}$ Yu T Zhao, ${ }^{1}$ Robert Ambrosini, ${ }^{1}$ Sean Cleary, ${ }^{1}$ Deborah Rubens, ${ }^{1}$ Mitchell Chess, ${ }^{1}$ Benjamin Castaneda, ${ }^{5}$ Ann Dozier, ${ }^{6}$ Timothy O'Connor, ${ }^{7}$ Brian Garra, ${ }^{8}$ Katherine Kaproth-Joslin ${ }^{1}$

\section{ABSTRACT}

Background Respiratory illness is a leading cause of morbidity in adults and the number one cause of mortality in children, yet billions of people lack access to medical imaging to assist in its diagnosis. Although ultrasound is highly sensitive and specific for respiratory illness such as pneumonia, its deployment is limited by a lack of sonographers. As a solution, we tested a standardised lung ultrasound volume sweep imaging (VSI) protocol based solely on external body landmarks performed by individuals without prior ultrasound experience after brief training. Each step in the VSI protocol is saved as a video clip for later interpretation by a specialist.

Methods Dyspneic hospitalised patients were scanned by ultrasound naive operators after 2 hours of training using the lung ultrasound VSI protocol. Separate blinded readers interpreted both lung ultrasound VSI examinations and standard of care chest radiographs to ascertain the diagnostic value of lung VSI considering chest X-ray as the reference standard. Comparison to clinical diagnosis as documented in the medical record and CT (when available) were also performed. Readers offered a final interpretation of normal, abnormal, or indeterminate/borderline for each VSI examination, chest X-ray, and CT.

Results Operators scanned 102 subjects (0-89 years old) for analysis. Lung VSI showed a sensitivity of $93 \%$ and a specificity of $91 \%$ for an abnormal chest X-ray and a sensitivity of $100 \%$ and a specificity of $93 \%$ for a clinical diagnosis of pneumonia. When any cases with an indeterminate rating on chest $\mathrm{X}$-ray or ultrasound were excluded $(n=38)$, VSI lung ultrasound showed $92 \%$ agreement with chest X-ray (Cohen's $\kappa 0.83$ (0.68 to $0.97, p<0.0001))$. Among cases with CT $(n=21)$, when any ultrasound with an indeterminate rating was excluded $(n=3)$, there was $100 \%$ agreement with VSI.

Conclusion Lung VSI performed by previously inexperienced ultrasound operators after brief training showed excellent agreement with chest $X$-ray and high sensitivity and specificity for a clinical diagnosis of pneumonia. Blinded readers were able to identify other respiratory diseases including pulmonary oedema and pleural effusion. Deployment of lung VSI could benefit the health of the global community.

For numbered affiliations see end of article.

Correspondence to Dr. Thomas J Marini; rochesterradiology2021@ gmail.com

\section{INTRODUCTION}

Pneumonia is the leading cause of death in children aged $<5$ years with hundreds

\section{Key messages}

We questioned whether operators without significant ultrasound background can obtain diagnostic imaging of the lungs by following a standardised volume sweep imaging (VSI) ultrasound protocol based on external body landmarks to detect respiratory diseases such as pneumonia, pulmonary edema, and pleural effusion.

- Operators without prior ultrasound experience can obtain diagnostic images of respiratory disease including pneumonia, pulmonary edema, and pleural effusion on par with standard of care imaging.

- Respiratory illness is a major cause of morbidity and mortality around the world. Pneumonia is the leading cause of death among children aged $0-5$ years with nearly 1 million deaths per year. Lung ultrasound VSI could assist in expanding access to respiratory imaging in low-resource settings and potentially improve disparities in global health.

of thousands of deaths reported annually. ${ }^{2}$ Yearly, approximately 150 million children between the ages of 0 and 5 years develop pneumonia with up to 20 million of these cases requiring hospitalisation. ${ }^{3}$ In adults, respiratory illnesses such as chronic obstructive pulmonary disease (COPD), asthma, infection, and lung cancer remain prevalent with chronic respiratory disease a leading cause of morbidity worldwide. ${ }^{4-6}$ Clinical history and physical examination do not reliably differentiate between causes of respiratory illnesses, as the signs and symptoms (such as fever and cough) are generally non-specific. ${ }^{78}$ Thus, reliable diagnosis often requires medical imaging, but billions of people (two-third of the world) lack access to diagnostic imaging presumably worsening outcomes related to respiratory illness. ${ }^{9-11}$

Ultrasound has emerged as a promising imaging modality to deliver diagnostic pulmonary imaging to underserved 
areas. ${ }^{12}{ }^{13}$ Lung ultrasound has shown excellent sensitivity and specificity for pneumonia in adults and children, consistently showing $>90 \%$ sensitivity and specificity in meta-analysis. ${ }^{14}{ }^{15}$ Lung ultrasound also accurately assists in evaluation of pleural effusion and pulmonary oedema with similar accuracy. ${ }^{16-18}$ Current telemedicine technology is sufficient to perform lung ultrasound interpretation over low bandwidth removing one barrier to deployment in rural areas. ${ }^{19}{ }^{20}$ However, while telemedicine is feasible and ultrasound machines may be relatively inexpensive, training experienced personnel can be costly and time consuming.

Volume sweep imaging (VSI) is an ultrasound imaging technique that can be performed without an ultrasound professional as it utilises standardised imaging sweeps of the probe based solely on external body landmarks. ${ }^{13} 20$ This approach allows novices without significant medical knowledge or technical skill to acquire acceptable images after brief training. Each ultrasound probe sweep of the VSI protocol is saved as a cine clip for later review by an imaging specialist. Although VSI has similarities to point-of-care ultrasound (POCUS) which has already been shown to be easily learnt and highly accurate, there are critical differences. ${ }^{21} 22$ A key distinction is that VSI completely separates image acquisition from image interpretation. Furthermore, while POCUS is often tailored to the specific clinical scenario, VSI is a standardised approach that will be performed the same way each examination.

Although lung VSI theoretically promises to reliably diagnose pneumonia and other respiratory conditions, there have been no clinical studies of its diagnostic accuracy. In this study, we aimed to test a VSI lung ultrasound protocol in comparison with standard of care chest radiography, the clinical diagnosis documented in the electronic medical record, and chest CT when available. We hypothesised that lung VSI would yield interpretations in agreement with both standard of care radiography, clinical diagnosis, and chest CT scan.

\section{METHODS}

Study design

We undertook a prospective study to test a VSI lung ultrasound protocol at the University of Rochester Medical Center from May 2019 to January 2020. The medical centre's institutional review board reviewed and approved this study. Patients admitted to the hospital with a chest X-ray were scanned with the VSI lung ultrasound protocol on a Logiq E9 or Logiq E10 ultrasound machine (General Electric, Boston, USA). Consecutive eligible adult and paediatric patients during set scanning times were identified at the time of standard of care imaging by the radiology department. Inclusion criteria included standard of care imaging (chest X-ray) in the past week and shortness of breath. Patients with haemodynamic instability or a body mass index $(\mathrm{BMI})>40$ were excluded.
Following informed consent, a medical student or resident blinded to the chest X-ray results and history scanned subjects with the VSI protocol. To reduce any bias, the operator conducting the scan was explicitly instructed not to look at the screen during the VSI acquisitions. Each operator was shown how to use the ultrasound machine and perform the VSI scan protocol within 2 hours; he or she practiced the protocol 10 times independently prior to scanning patients. Following completion of the VSI protocol, cine clips were saved in MP4 format for later review. Clinical information from each patient's chart including demographic information, medical history, and clinical course were recorded. The clinical team was not informed of the lung ultrasound results.

\section{Lung VSI}

VSI is an imaging technique developed to increase the availability of imaging in underserved areas around the world. VSI protocols for obstetrics, right upper quadrant, and thyroid have previously been tested with encouraging results. ${ }^{20}{ }^{23-25}$ VSI generally employs straight line imaging sweeps of the ultrasound probe to cover the target region using only external body landmarks. Although experienced sonographers require extensive training to hone technical skills and anatomical knowledge, VSI can be performed after a few hours of training by individuals without significant medical experience. ${ }^{13}{ }^{20}$ Each sweep in a VSI protocol is saved as a cine clip for later interpretation by a specialist. It is important to emphasise that the person acquiring the images is not responsible for any imaging interpretation, a key difference when compared with POCUS. The entire VSI examination usually takes $10 \mathrm{~min}$ and is performed using machine presets obviating the need for technical adjustments.

The lung VSI protocol was designed in accordance with international consensus recommendations on the use of lung ultrasound..$^{26}$ Transverse and sagittal acquisitions are obtained in each lung field for redundancy and to better characterise findings. ${ }^{27}$ Rural workers in Peru learnt the protocol in about an hour and demonstrated error-free performance after only a few trials. ${ }^{13}$ The VSI protocol is shown schematically in figure 1 and in online supplemental video 1 . It consists of simple straight line sweeps along with arcs (fanning) of the probe to maximise visibility of the anterior, lateral, and posterior lung fields. Tips, pitfalls, and commentary on lung VSI training and performance have been previously described. ${ }^{13}$ One previously described lung POCUS protocol has shown $>90 \%$ diagnostic accuracy for pneumonia, pleural effusion, pulmonary oedema, pneumothorax, COPD, and asthma examining only three isolated sites in each hemithorax. ${ }^{21}$ As VSI is a more thorough examination, the theoretical diagnostic yield would be expected to be at least comparable. 


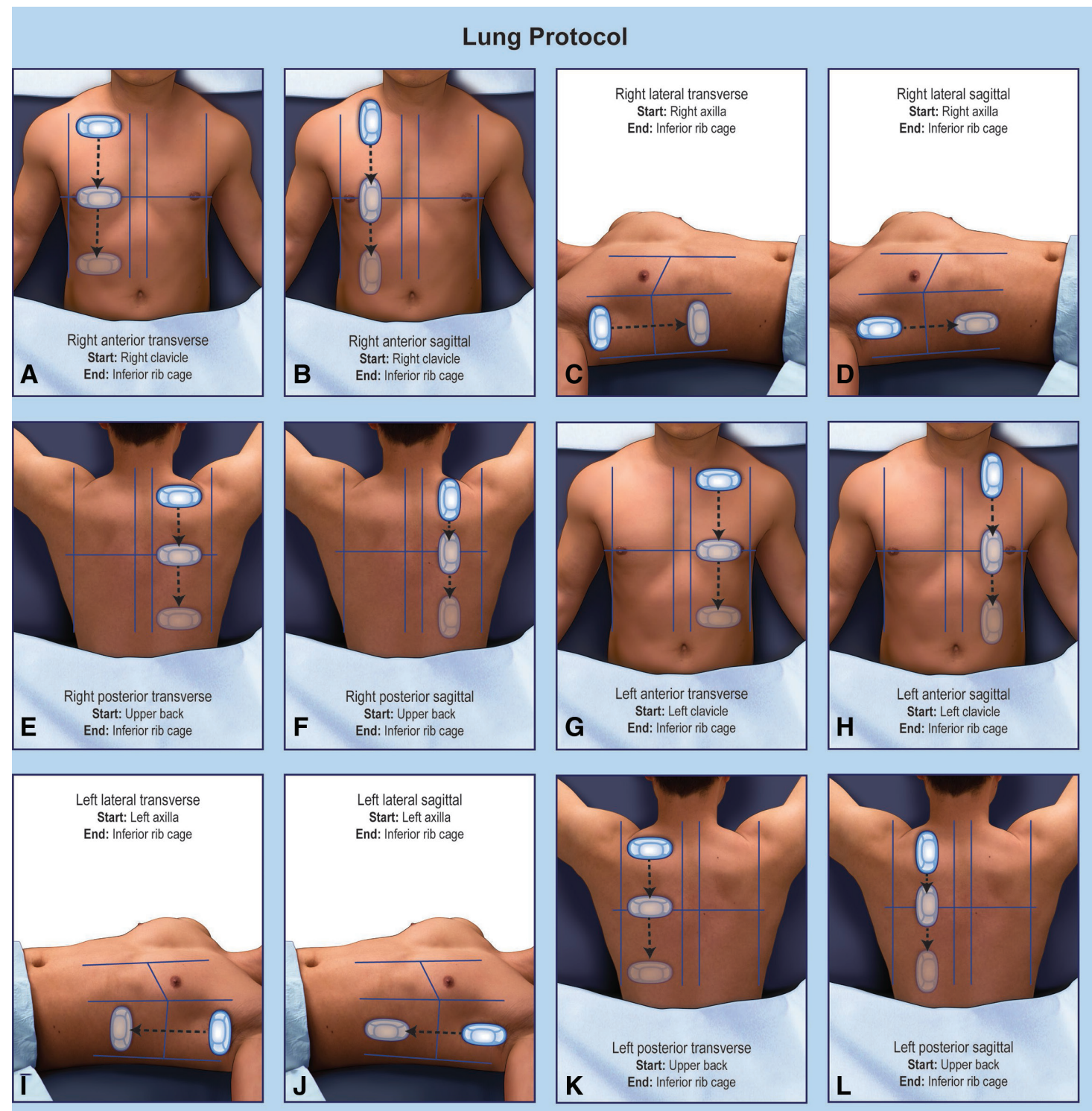

Figure 1 Lung VSI protocol. Poster illustrating the lung VSI protocol. Arcs are performed at each probe position shown on the diagram (superior, middle and inferior positions on the anterior and posterior lungs and superior and inferior positions on the lateral lungs). VSI, volume sweep imaging.

\section{Reference standards}

Three different reference standards were used in this study, each with its own benefits and drawbacks. The primary reference standards were standard of care chest radiography and the clinical diagnosis as noted in the medical record. The clinical diagnosis was extracted from the medical record as written in the history of present illness/discharge summary. When $>1$ diagnosis was specified or a differential was given, the clinical diagnosis was noted as indeterminate. In a subset of patients, CT scan also served as a third reference standard when performed.

\section{Blinded readings}

Randomised blinded readings of the chest X-rays, lung VSI clips, and chest CTs (when available) were performed. Two cardiothoracic radiologists with 2 and 4 years of experience interpreted all standard of care chest X-rays. Chest CTs were randomised and interpreted by these same radiologists at a later date independent of radiograph interpretation. A cardiothoracic radiologist (6 years of experience) and a critical care pulmonologist (9 years of experience) interpreted the VSI lung ultrasound studies. The original diagnostic chest X-ray and chest CT reports were not used for analysis.

\section{Definitions}

Examinations were classified as abnormal, normal, or indeterminate/borderline by all interpreters removing ambiguity in assessing agreement from free-text descriptions and situations involving multifocal abnormalities. The location of the abnormality was not specified in 
the final analysis. Readers were asked to call something abnormal or normal only if they were $>90 \%$ certain of their interpretation. Pneumonia, pleural effusion, and pulmonary oedema were all rated as abnormal. Negative studies or studies with minimal expected atelectasis or emphysema were rated as normal. The purpose of the indeterminate/borderline category was to avoid arbitrary disagreement or agreement secondary to truly ambiguous findings. This category was used when a reader felt unable to offer a conclusive rating of definitively normal or abnormal. For example, a lower lung opacity considered indeterminate for infection or atelectasis was noted as indeterminate/borderline.

Individual blinded readings were analysed for interreader reliability. Any discrepant interpretations were reviewed, and a final consensus read was decided for each ultrasound, radiograph, and CT. For each examination, the reader also wrote free text notes on image quality and findings. As VSI is a relatively new imaging technique and reading VSI examinations is a different experience from regular clinical practice, 10 random subjects were removed for a training set prior to blinded readings. The readers went through the training set per normal study procedures and then were unblinded to the radiographic or ultrasound findings to acclimate themselves to the study and VSI.

\section{Patient and public involvement}

Patients and the public were not involved in the formulation of this research study outside of serving as our research subjects. However, the study was designed based on our prior experiences working with rural communities in Peru with the goal of generalising our research findings to support underserved communities around the world. Dissemination of these results will be targeted to improve the health of underserved communities.

\section{Statistical analysis}

Group comparisons were performed using ordinary one-way ANOVA for continuous measures and the $\chi^{2}$ test for categorical variables. Inter-reader agreement was evaluated for ultrasound and X-ray independently using Cohen's $\kappa$, with an additional comparison between consensus ultrasound and consensus X-ray interpretation. Sensitivity, specificity, positive predictive value (PPV), negative predictive value (NPV), and positive/ negative likelihood ratios were calculated for ultrasound with the blinded consensus read of the standard of care radiograph as the reference standard, as well as for both ultrasound and chest X-ray with clinical diagnosis as documented in the medical record as the reference standard. Sensitivity and specificity were compared between imaging modalities using McNemar's test. Multivariate analysis was performed using logistic regression with consensus ultrasound as the predictor, age, sex, worst temperature, and worst oxygen saturation as covariates, and either abnormal consensus X-ray or clinical diagnosis of pneumonia as the outcome. For both regression and calculation of sensitivity, specificity, PPV and NPV, indeterminate/borderline cases were excluded from analysis. All statistical analysis was performed in MATLAB 2019b (The Mathworks, Natick, MA, USA) and SPSS V.26 (IBM Corp., Armonk, NY, USA).

\section{RESULTS}

A total of 102 subjects were used for analysis (62 paediatric subjects and 40 adult subjects) from the original 112 subjects enrolled (10 removed for the training set). There were no adverse events related to the performance of lung ultrasound. The readers found all VSI examinations and radiography to be of diagnostic utility with acceptable imaging quality. On average, sweeps took $17-25$ s to perform with the total recorded imaging time averaging $4.3 \mathrm{~min} .97 \%$ of ultrasound examinations were scanned within 72 hours of chest X-ray acquisition. Online supplemental table 1 shows overall study subject characteristics.

\section{Lung VSI compared with chest X-ray}

Ultrasound results compared with consensus chest X-ray rating are shown in table 1 for all subjects, online supplemental table 2 for paediatric subjects, and online supplemental table 3 for adult subjects. Among all subjects with an abnormal chest X-ray, $80 \%$ of the ultrasounds were read as abnormal, $6 \%$ normal, and $14 \%$ indeterminate/ borderline. For all subjects with a normal chest X-ray, $77 \%$ of ultrasounds were read as normal, $8 \%$ abnormal, and $15 \%$ indeterminate/borderline. In the paediatric population, among clinical variables analysed, the only significant differences found between abnormal, indeterminate/borderline, and normal chest X-rays were antibiotic administration $(\mathrm{p}=0.026)$, greatest oxygen supplementation $(\mathrm{p}=0.0017)$, and lung ultrasound $(\mathrm{p}<0.0001)$. This analysis was not performed for adults due to the small sample of normal adult studies $(n=3)$.

\section{Lung VSI, chest X-ray, and chest CT compared with clinical diagnosis}

Subject characteristics compared with clinical diagnosis as noted in the electronic medical record are shown in table 2 for all subjects, online supplemental table 4 for paediatric subjects, and online supplemental table 5 for adult subjects. Among all subjects, lung ultrasound performed on par with chest X-ray with lung ultrasound identifying pneumonia as abnormal $95 \%$ of the time. Chest X-ray identified pneumonia as abnormal $92 \%$ of the time. Among those with asthma or non-respiratory syncytial virus (non-RSV) viral illness, $94 \%$ of lung ultrasound examinations were rated normal or indeterminate/borderline (81\% normal and $13 \%$ indeterminate/ borderline). Chest X-ray also identified $94 \%$ of these examinations as normal or indeterminate/borderline. RSV positive viral illness produced a mix of results among all categories for both chest X-ray and lung ultrasound 

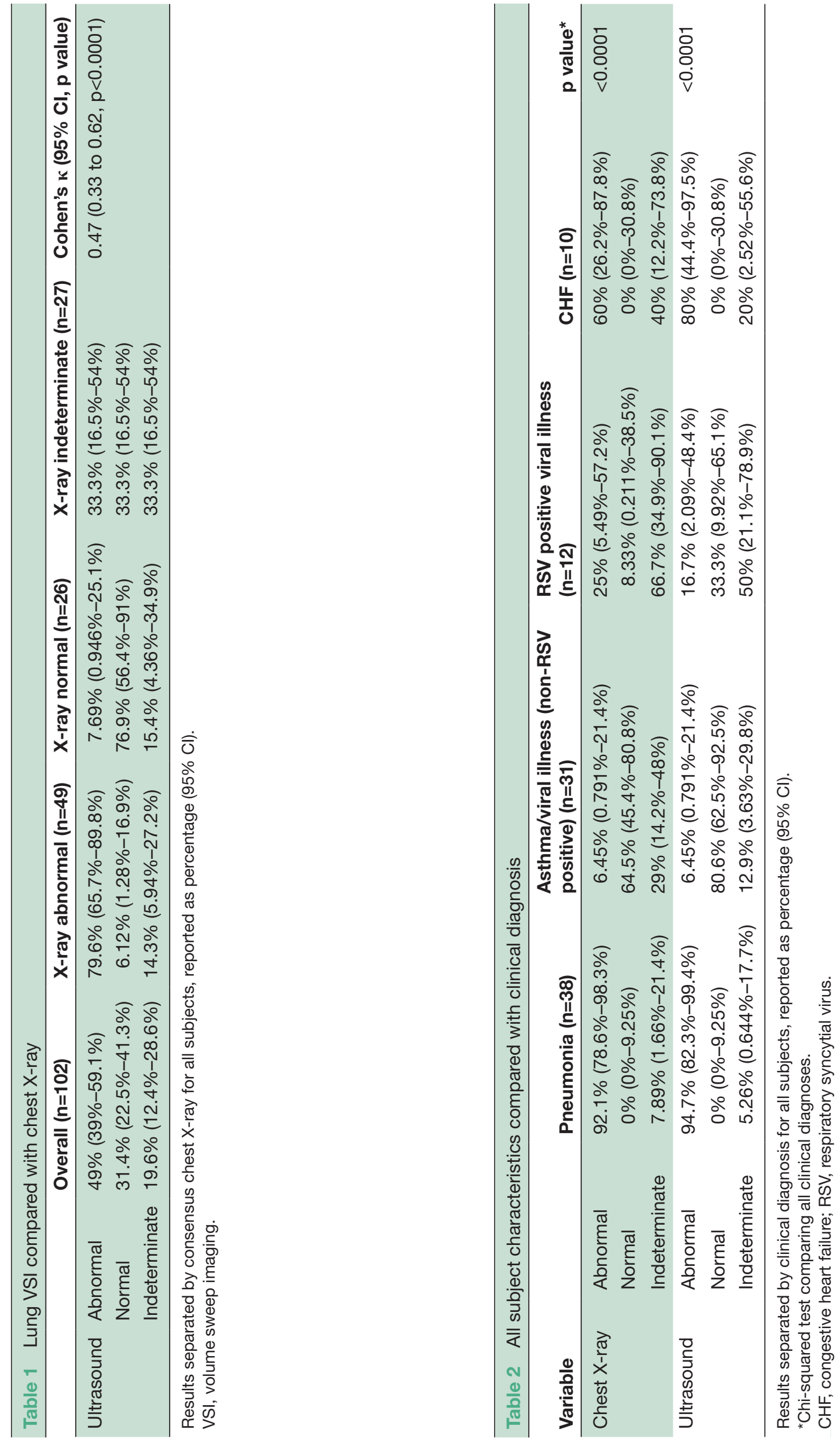

윰 
Table 3 Reader agreement

\begin{tabular}{|c|c|c|c|c|}
\hline \multirow[b]{2}{*}{ Comparison } & \multicolumn{2}{|c|}{$\begin{array}{l}\text { Agreement without indeterminate/ } \\
\text { borderline cases }\end{array}$} & \multicolumn{2}{|c|}{$\begin{array}{l}\text { Agreement including indeterminate } \\
\text { borderline cases }\end{array}$} \\
\hline & Agreement & Cohen's к (95\% Cl, p value) & Agreement & $\begin{array}{l}\text { Cohen's к (95\% Cl, p } \\
\text { value) }\end{array}$ \\
\hline Ultrasound inter-reader agreement & $91.4 \%$ & $0.82(0.69$ to $0.96, p<0.0001)$ & $69.6 \%$ & $\begin{array}{l}0.52 \text { ( } 0.38 \text { to } 0.66 \\
p<0.0001)\end{array}$ \\
\hline Chest X-ray inter-reader agreement & $96.9 \%$ & 0.93 (0.84 to $1, p<0.0001)$ & $77.5 \%$ & $\begin{array}{l}0.65 \text { (0.53 to } 0.78 \\
\mathrm{p}<0.0001)\end{array}$ \\
\hline $\begin{array}{l}\text { Consensus ultrasound vs consensus } \\
\text { chest X-ray agreement }\end{array}$ & $92.2 \%$ & 0.83 (0.68 to $0.97, p<0.0001)$ & $66.7 \%$ & $\begin{array}{l}0.47(0.33 \text { to } 0.62 \\
p<0.0001)\end{array}$ \\
\hline
\end{tabular}

Inter-reader agreement for ultrasound and X-ray separately and between consensus ultrasound and X-ray. The agreement without indeterminate/borderline cases was calculated from those in which no indeterminate/borderline ratings were recorded.

with indeterminate/borderline ratings predominating. For congestive heart failure $(\mathrm{CHF}), 80 \%$ of lung ultrasounds were rated abnormal and 20\% indeterminate/ borderline compared with chest X-ray showing $60 \%$ abnormal and $40 \%$ indeterminate/borderline. CT agreed with the clinical diagnosis in all cases with 15 cases of pneumonia, 5 cases of CHF, and 1 case of COPD.

\section{Lung ultrasound and chest X-ray compared with CT}

CT was also obtained in $\mathrm{n}=21$ cases, which were overwhelmingly abnormal $(n=20)$ when sorted by the CT consensus read. There was only one normal/negative CT scan corresponding to a clinical diagnosis of COPD. When all indeterminate/borderline scans were excluded among ultrasound and CT ( $\mathrm{n}=3)$, there was $100 \%$ agreement between lung ultrasound and CT imaging. Chest $\mathrm{X}$-ray showed similar results, with $100 \%$ agreement when any case with an indeterminate/borderline rating was excluded $(n=3)$.

\section{Lung ultrasound and chest X-ray agreement}

There was excellent inter-reader agreement and agreement between chest X-ray and ultrasound when cases with any indeterminate/borderline rating between ultrasound and chest X-ray were removed from analysis, with a Cohen's $\kappa$ of $0.83(0.68-0.97, \mathrm{p}<0.0001)$ for agreement between modalities (table 3). When considering the indeterminate/borderline category, there were 35 cases with any level of disagreement between chest X-ray and ultrasound. Of this number, 30 cases involved the use of an indeterminate/borderline rating in either the ultrasound or the chest X-ray. Most indeterminate/borderline cases on ultrasound were related to small subpleural consolidations or B-lines of indeterminate pathological significance. Most indeterminate/borderline chest X-ray cases involved subtle opacities that were non-specific.

Of the remaining five cases where one modality was rated as normal and the other as abnormal, explanations for the discordance varied. In one of these cases, blinded VSI readers mistook the spleen for consolidation, a potential pitfall to VSI examination correctable with experience. Two other cases related to viral illnesses that ultrasound called normal and chest X-ray called abnormal. The final two cases were clinically ambiguous without a clear aetiology of disagreement.

The sensitivity and specificity of lung ultrasound in this study was not significantly different from chest X-ray for a clinical diagnosis of pneumonia ( $p>0.99$ for sensitivity and $\mathrm{p}=0.48$ for specificity) (online supplemental table 6 ). Lung ultrasound VSI was $93 \%$ sensitive and $91 \%$ specific for an abnormal chest X-ray and $100 \%$ sensitive and $93 \%$ specific for pneumonia. Multivariate logistic regression to identify predictors of an abnormal chest X-ray and a clinical diagnosis of pneumonia among the consensus lung ultrasound interpretation, age, sex, worst temperature, and worst oxygen saturation showed consensus lung ultrasound as the only statistically significant predictor for both an abnormal chest X-ray $(p=0.026)$ and a clinical diagnosis of pneumonia $(\mathrm{p}<0.0001)$ (online supplemental table 7).

\section{DISCUSSION}

The lung ultrasound VSI protocol performed by previously ultrasound-naive operators showed statistically significant agreement with radiography and the clinical diagnosis. There were no significant differences in sensitivity or specificity between VSI lung ultrasound and standard of care radiography for pneumonia as documented in the electronic medical record. Among the cases with a CT correlate, there was $100 \%$ agreement with VSI lung ultrasound when the three indeterminate ultrasound cases were excluded from analysis. Readers of the VSI cine clips found acceptable imaging quality despite imaging being obtained by those without prior ultrasound experience after brief training. These findings suggest that VSI lung ultrasound is an effective imaging technique at least on par with chest X-ray. In rural areas without access to high-quality imaging, implementation of VSI could potentially offer an accurate, low-cost means for diagnosis of respiratory disease.

A general review of radiographic findings suggests that our patients were sufficiently diverse to capture 


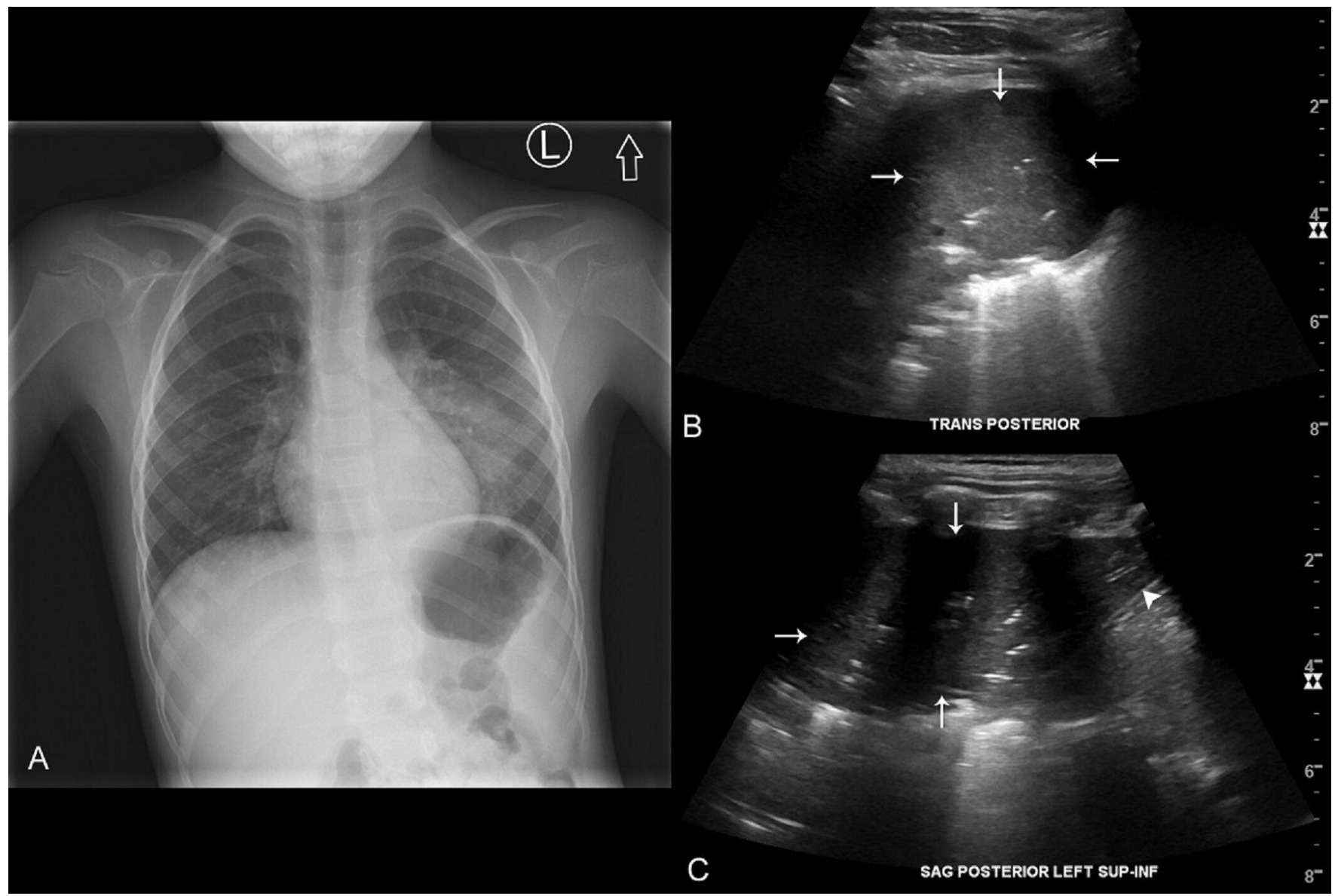

Figure 2 Pneumonia on VSI. (A) Chest X-ray of a 5-year-old male with a left lower lobe pneumonia. Single-frame (B) transverse and $(\mathrm{C})$ sagittal views of the patient's left lower pneumonia (arrows) obtained during the left posterior sweeps. (C) The diaphragm (arrowhead) is seen. A VSI cine clip has been submitted as Video 2. VSI, volume sweep imaging.

the spectrum of pathology ranging from simple viral illness to life threatening pneumonia. Imaging examples including pneumonia, pleural effusion, vaping-induced lung injury, and bronchiolitis are shown in figure 2 and online supplemental figures 1-3 with corresponding VSI cine clips provided in online supplemental videos $2-5$. In this study, VSI did not miss any cases of clinically acute pneumonia suggesting that a normal VSI examination may be able to rule out clinically significant pneumonia. Lung VSI also successfully visualised heart failure and pleural effusion and was appropriately negative in COPD, asthma, and non-RSV viral illness. Viral illness in children $<2$ years of age poses a special challenge for both lung ultrasound and X-ray due to variable imaging findings and overlapping appearance of atelectasis and infection. Two-third of our patients with RSV had indeterminate/borderline chest X-rays and $50 \%$ had indeterminate/borderline ultrasound examinations. It has been previously shown that bronchiolitis, in many cases, can be difficult to distinguish from pneumonia. ${ }^{28-30}$ In these cases, a normal ultrasound is worthwhile as it precludes antibiotic therapy.

The utilisation of an indeterminate/borderline rating was used in this study to avoid arbitrary agreement and disagreement in ambiguous cases, as many cases in true clinical practice have equivocal findings that require clinical correlation. It also allowed for a rough stratification of cases by their severity. If a reader was uncertain as to whether a finding was abnormal or normal, it may be due to debatable clinical relevance. When readers interpreted a study as definitively normal or abnormal, this was almost always in agreement with the other modality with only 5 cases of disagreement in the study. Thus, pathology like lobar pneumonia, which is both clinically significant and potentially life-threatening, is expected to be detected without overlap with atelectasis or other indeterminate findings. Furthermore, these studies were interpreted without any clinical context, and in real-life practice, findings should be correlated to the patient's clinical history and examination.

This imaging technique is ideal for use in low-resource and underserved areas and already fits into existing telediagnostic infrastructure previously published incorporating VSI. ${ }^{20}$ A proposed model for lung VSI using this asynchronous telediagnostic system is shown in online supplemental figure 4 . The proposed integration allows imaging to be delivered to rural and underserved communities for the price of a tablet and portable ultrasound machine, removing substantial barriers to imaging access. In practice, VSI examination can be performed as 
often as necessary to monitor patients (especially useful in indeterminate cases) as it is inexpensive and requires no radiation exposure.

Additional ancillary benefits to the deployment of this approach that extend beyond the population health benefits of timely diagnosis and appropriate treatment may be realised by increased clinic attendance. For example, when VSI obstetric ultrasound was deployed in Uganda, more people attended clinic and received prenatal testing. ${ }^{31}$ Among other benefits, lung VSI deployment could result in increased vaccination rates and patient education. Even outside rural and developing countries, the potential uses of VSI are numerous and impact public health. An ultrasound novice trained on the VSI protocol could be stationed in a busy Emergency Department for minimal cost to triage patients prior to clinical examination. This could potentially assist in diagnosis and treatment planning while simultaneously improving turnaround times, decreasing X-ray utilisation, and minimising radiation exposure to children.

Establishing the best reference standard for the VSI studies was complicated with both chest X-ray and the clinical diagnosis identified in the medical record having drawbacks. Chest CT is an ideal reference standard but was only available in a subset of patients resulting in a biased sample. We used all three reference standards to give the most comprehensive analysis possible. In the same vein, adult and paediatric patients were used in this study to maximise generalisability at the tradeoff of increased heterogeneity of cases. In particular, analysis of clinical variables across these populations was limited secondary to the inherent differences in each age group. Nonetheless, overlap in the imaging findings of both adults and children justified their pooling in our clinical analysis to increase statistical power, and supplemental tables have been provided to better delineate the relationship of the clinical variables to the findings without confounding by age. As the goal of this study was to establish a proof-of-concept, the limitations associated with the heterogeneity of the sample do not significantly limit our conclusions.

For logistical reasons, this study was performed in a high-resource hospital. Partially, this was to allow access to the electronic medical record along with reference standard imaging often not readily available in the communities where this approach would be deployed. Similarly, to comply with institutional review board regulations, the ultrasound operators in this study were a radiology resident and six medical students without prior lung ultrasound experience. Although this introduced possible bias from their medical knowledge, these operators were blinded to the clinical condition and explicitly instructed not to look at the ultrasound screen. In addition, previous study has shown rural workers in Peru learnt the scanning protocol without difficulty. ${ }^{13}$ Nonetheless, future studies should be aimed at replicating these findings in rural areas with operators with less medical background.
An additional limitation of the study was our exclusion of hemodynamically unstable patients. In general, those with life-threatening respiratory illness tend to have more obvious findings on ultrasound as a function of the severity of their illness but were less likely to enroll. If scans included more critically ill patients, VSI would theoretically show even better diagnostic accuracy, as life-threatening conditions tend to be more prominent on imaging. ${ }^{32}$ Patients with a BMI $>40$ were not included in this study which also somewhat limits generalisability. Although VSI can be attempted in these patients and may provide some utility, like other ultrasound examinations, increased body habitus may limit imaging quality in this population.

Millions of people around the world lack access to diagnostic imaging for evaluation of respiratory illnesses. Closing the gap in the vast disparities in healthcare delivery is a moral imperative for the medical profession. Lung ultrasound VSI combined with teleultrasound offers a promising low-cost means to diagnose many respiratory conditions including infection, pulmonary oedema, and pleural effusion without an experienced sonographer. Deployment of lung VSI holds the potential to improve imaging access to underserved communities, improving the health and well-being of the global community.

Author affiliations

${ }^{1}$ Department of Imaging Sciences, URMC, Rochester, NY, USA

${ }^{2}$ Department of Medicine, URMC, Rochester, NY, USA

${ }^{3}$ University of Rochester School of Medicine and Dentistry, URMC, Rochester, NY, USA

${ }^{4}$ Department of Medicine, Boston University Medical Center, Boston, MA, USA ${ }^{5}$ Departmento de Ingeniería, Pontificia Universidad Católica del Perú, Lima, Peru

${ }^{6}$ Department of Public Health Sciences, URMC, Rochester, NY, USA ${ }^{7}$ Department of Emergency Medicine, URMC, Rochester, NY, USA

${ }^{8}$ Medical Imaging Ministries of the Americas, Clermont, FL, USA

Acknowledgements We graciously thank all the patients and their families who participated in this study. We would like to thank Dr. Thomas Foster, Lisa Boisvert, Jannette Cong, Matthew Smith and the Fischer Fund Committee for their assistance in the funding of this research. We would like to thank Laurie Christensen, Erica Longbine, and JoAnne McNamara for their assistance as study coordinators. We would like to thank Nadezhda Kiriyak, Sarah Klingenberger, Jane Lichorowic, Gwen Mack, and Dr. Solomon Kim for their assistance in preparing the figures and illustrations. We would like to thank Dr. David Waldman, Dr. Susan Hobbs, Dr. Nina Klionsky, Dr. Apeksha Chaturvedi, and Dr. Kevin Parker for their support and mentorship. We would like to thank Tracy Apeland RDMS Nancy Carson RDMS, and Chelsea Pino RDMS for their sonographic expertise and support while conducting this study. We would like to thank Walter Sifuentes, Claudia Carlotto, Mauricio Salazar, Lorena Tamayo, and Medical Innovation and Technology for allowing us to publish the lung VSI training video as supplemental material.

Contributors All authors made substantial contributions to the paper. All authors provided final approval of the version to be published and agree to be accountable for all aspects of the work. KKJ serves as the guarantor for the work

Funding University of Rochester Fischer Fund (Testing a VSI Protocol for Pneumonia Detection). Institutional funding was provided for the scanning of patients at our institution. The funding committee had no input in the study or manuscript design.

Competing interests Benjamin Castaneda has financial stake in the company Medical Innovation and Technology which seeks to bring ultrasound to rural communities. The other authors declare no conflict of interest.

Patient consent for publication Consent obtained directly from patient(s) 
Ethics approval University of Rochester Research Subjects Review Board. Study 00004066.

Provenance and peer review Not commissioned; externally peer reviewed.

Data availability statement Data are available upon reasonable request.

Supplemental material This content has been supplied by the author(s). It has not been vetted by BMJ Publishing Group Limited (BMJ) and may not have been peer-reviewed. Any opinions or recommendations discussed are solely those of the author(s) and are not endorsed by BMJ. BMJ disclaims all liability and responsibility arising from any reliance placed on the content. Where the content includes any translated material, BMJ does not warrant the accuracy and reliability of the translations (including but not limited to local regulations, clinical guidelines, terminology, drug names and drug dosages), and is not responsible for any error and/or omissions arising from translation and adaptation or otherwise

Open access This is an open access article distributed in accordance with the Creative Commons Attribution Non Commercial (CC BY-NC 4.0) license, which permits others to distribute, remix, adapt, build upon this work non-commercially, and license their derivative works on different terms, provided the original work is properly cited, appropriate credit is given, any changes made indicated, and the use is non-commercial. See: http://creativecommons.org/licenses/by-nc/4.0/.

ORCID iD

Thomas J Marini http://orcid.org/0000-0002-1529-5097

\section{REFERENCES}

1 Walker CLF, Rudan I, Liu L, et al. Global burden of childhood pneumonia and diarrhoea. Lancet 2013;381:1405-16.

2 Wardlaw T, Salama P, Johansson EW, et al. Pneumonia: the leading killer of children. Lancet 2006;368:1048-50.

3 Rudan I, Tomaskovic L, Boschi-Pinto C, et al. Global estimate of the incidence of clinical pneumonia among children under five years of age. Bull World Health Organ 2004;82:895-903.

4 Forum of International Respiratory Societies. The global impact of respiratory disease -. Second Edition, 2017.

5 Avendaño Carvajal L, Perret Pérez C. Epidemiology of Respiratory Infections. In: Pediatric respiratory diseases: a comprehensive textbook. Cham: Springer International Publishing, 2020: 263-72.

6 Xie M, Liu X, Cao X, et al. Trends in prevalence and incidence of chronic respiratory diseases from 1990 to 2017. Respir Res 2020;21:49.

7 Wipf JE, Lipsky BA, Hirschmann JV, et al. Diagnosing pneumonia by physical examination: relevant or relic? Arch Intern Med 1999:159:1082-7.

8 Kelsberg G, Safranek S, Ely JW. Clinical inquiries. How accurate is the clinical diagnosis of pneumonia? J Fam Pract 2003;52:63-4.

9 Pan American Health Organization. World Radiography Day: Two-Thirds of the World's Population has no Access to Diagnostic Imaging. Available: https://www.paho.org/hq/index.php?option= com content\&view=article\&id=7410:2012-dia-radiografia-dostercios-poblacion-mundial-no-tiene-acceso-diagnostico-imagen\& Itemid=1926\&lang=en\#: :text=\%2D\%20The\%20use\%20of\%20X\% 2Drays,no\%20access \%20to\%20diagnostic\%20imaging.\&text=It\% 20 is\%20used\%20for\%20diagnostic\%2C\%20preventive\%2C\% 20and\%20therapeutic\%20purposes [Accessed 10/30/2020].

10 Mollura DJ, Mazal J, Everton KL, et al. White paper report of the 2012 RAD-AID conference on international radiology for developing countries: planning the implementation of global radiology. J Am Coll Radiol 2013;10:618-24.

11 Ngoya PS, Muhogora WE, Pitcher RD. Defining the diagnostic divide: an analysis of registered radiological equipment resources in a low-income African country. Pan Afr Med J 2016;25:99.
12 Amatya Y, Rupp J, Russell FM, et al. Diagnostic use of lung ultrasound compared to chest radiograph for suspected pneumonia in a resource-limited setting. Int J Emerg Med 2018;11:8.

13 Marini TJ, Castaneda B, Baran T, et al. Lung ultrasound volume sweep imaging for pneumonia detection in rural areas: Piloting training in rural Peru. J Clin Imaging Sci 2019;9:35.

14 Pereda MA, Chavez MA, Hooper-Miele CC, et al. Lung ultrasound for the diagnosis of pneumonia in children: a meta-analysis. Pediatrics 2015; $135: 714-22$

$15 \mathrm{Ye} \mathrm{X}$, Xiao H, Chen B, et al. Accuracy of lung ultrasonography versus chest radiography for the diagnosis of adult community-acquired pneumonia: review of the literature and meta-analysis. PLoS One 2015;10:e0130066.

16 Lichtenstein D, Goldstein I, Mourgeon E, et al. Comparative diagnostic performances of auscultation, chest radiography, and lung ultrasonography in acute respiratory distress syndrome. Anesthesiology 2004;100:9-15.

17 Marini TJ, Rubens DJ, Zhao YT, et al. Lung ultrasound: the essentials. Radiol Cardiothorac Imaging 2021;3:e200564.

18 Maw AM, Hassanin A, Ho PM, et al. Diagnostic accuracy of point-of-care lung ultrasonography and chest radiography in adults with symptoms suggestive of acute decompensated heart failure: a systematic review and meta-analysis. JAMA Netw Open 2019;2: 190703.

19 Britton N, Miller MA, Safadi S, et al. Tele-Ultrasound in resourcelimited settings: a systematic review. Front Public Health 2019;7:244.

20 Marini TJ, Oppenheimer DC, Baran TM, et al. New ultrasound Telediagnostic system for low-resource areas: pilot results from Peru. J Ultrasound Med 2021;40:583-95.

21 Lichtenstein DA. BLUE-protocol and FALLS-protocol: two applications of lung ultrasound in the critically ill. Chest 2015;147:1659-70.

22 See $\mathrm{KC}$, Ong V, Wong SH, et al. Lung ultrasound training: curriculum implementation and learning trajectory among respiratory therapists. Intensive Care Med 2016;42:63-71.

23 Marini TJ, Weiss SL, Gupta A, et al. Testing telediagnostic thyroid ultrasound in Peru: a new horizon in expanding access to imaging in rural and underserved areas. J Endocrinol Invest 2021. doi:10.1007/ s40618-021-01584-7. [Epub ahead of print: 10 May 2021]

24 Toscano M, Marini TJ, Drennan K, et al. Testing telediagnostic obstetric ultrasound in Peru: a new horizon in expanding access to prenatal ultrasound. BMC Pregnancy Childbirth 2021;21:328

25 Marini TJ, Oppenheimer DC, Baran TM, et al. Testing telediagnostic right upper quadrant abdominal ultrasound in Peru: a new horizon in expanding access to imaging in rural and underserved areas. PLOS One 2021;16:e0255919.

26 Volpicelli G, Elbarbary M, Blaivas M, et al. International evidencebased recommendations for point-of-care lung ultrasound. Intensive Care Med 2012;38:577-91.

27 Milliner BHA, Tsung JW. Lung consolidation locations for optimal lung ultrasound scanning in diagnosing pediatric pneumonia. $J$ Ultrasound Med 2017;36:2325-8.

28 Biagi C, Pierantoni L, Baldazzi M, et al. Lung ultrasound for the diagnosis of pneumonia in children with acute bronchiolitis. BMC Pulm Med 2018;18:191.

29 Buonsenso D, Musolino AM, Gatto A, et al. Lung ultrasound in infants with bronchiolitis. BMC Pulm Med 2019;19:159.

30 Mauro AD, Ammirabile A, Quercia M. Acute bronchiolitis: is there a role for lung ultrasound? Diagnostics 2019:9:172.

31 Ross AB, DeStigter KK, Rielly M, et al. A low-cost ultrasound program leads to increased antenatal clinic visits and attended deliveries at a health care clinic in rural Uganda. PLoS One 2013;8:e78450.

32 Lichtenstein DA. Lung ultrasound in the critically ill. Ann Intensive Care 2014;4:1 